\title{
Transformation around intruders in granular media
}

\author{
Pierre Jop ${ }^{1, \star}$, Aymeric Merceron ${ }^{1}$, and Alban Sauret ${ }^{1}$ \\ ${ }^{1}$ Surface du Verre et Interfaces, UMR 125 CNRS/Saint-Gobain, 33 quai Lucien Lefranc, 93303 Aubervilliers, France
}

\begin{abstract}
Sintering, glass melting and other industrially relevant processes turn batches of grains into homogeneous products. Such processes involve coupled chemical and physical transformations of the granular packing. For sake of simplicity, we study how local evolutions on grains (volume decreases for example) entail mechanical rearrangements in the overall pile. Inert bidisperse metallic disks are mixed and confined in a vertical 2D cell. At the bottom of this set-up, initial intruders (one or two) have been previously set and mechanically linked to a linear motorized jack. While we quasi-statically pull the intruder(s) downward out of the cell at constant speed and constant liberated surface, we tracked the surrounding granular packing. Events largely distributed both spatially and temporally occur around intruders. We focus on the influence of the distance between the intruders on the local dynamic of the packing. We compare the distribution of the size of the event and their frequency as function of the relative position of the intruder, as a function of their radius. We show that their influence decreases rapidly. At short distance the mechanical perturbation induced by one intruder can destabilize the packing around the other.
\end{abstract}

\section{Introduction}

Sintering, glass melting and other industrially relevant processes turn batches of grains into homogeneous endproducts. Such processes lead to complex and mostly misunderstood phenomena due to chemical and physical transformations occurring inside the granular packing. Volume changes due to dilatation coefficients [1] or phase transitions may obviously affect the particles [2]. Consequently, the particle's shapes of particles evolves [3] as well as their contact properties through lubrication [4] or formation of capillary bridges [5]. In addition, chemical reactions also modify the surface and the nature of each individual grain [6]. These different phenomena occur at the same time and lead to the final homogeneous product through complex paths. Modeling the behavior of such systems generally involve different fields of research. Typically, mechanics and statistical physics are used to predict the behavior of granular assemblies under flow, rearrangement or perturbation [7]. The rich behavior of granular media has led to extensive studies considering their rheology [8], the criteria of mechanical stability [9] or the properties of wet granular assemblies [10]. Other fields of research has been considered to tackle such problems: chemical engineering where the behavior of reactive powders is modeled [11] and geology where rock transformations have been characterized by focusing on thermodynamics of reactions [12].

Some recent studies have characterized the rearrangement of a reactive granular packing, often icy particles than can melt $[2,13]$, but experimental investigations re-

\footnotetext{
^e-mail: pierre.jop@saint-gobain.com
}

main rare, likely because of the difficulties in monitoring the location of the individual grains as well as their properties. Therefore, most studies have focused on granular flows around an unique intruder using a mechanical approach [14-16], rheological approach [17] or a purely kinematic point of view $[18,19]$. Besides, much attention has been paid to the understanding of flows in silos and hoppers [20] through the arching problem [21] or for mining applications for which the grains are quasi-statically extracted [22].

In this paper we present experimental results on the response of bidimensional granular medium when two initial intruders are pulled out downward. These intruders are analog of melting grains that will disappear progressively. The $2 \mathrm{D}$ situation allows us to accurately characterize the position of each grain during the rearrangement process under gravity. We show that the behavior around an intruder is influenced by the other depending on their separation.

\section{A bidimensional reactive pile}

The reorganization of the granular medium around two intruders has been studied using a two-dimensional system (Fig. 1). The vertical cell is made of two glass plates (30 $\mathrm{cm}$ height and $60 \mathrm{~cm}$ width), separated by a gap of $3.1 \mathrm{~mm}$. The cell is filled with 5000 bi-disperse metallic disks of 4 and $5 \mathrm{~mm}$ in diameter (proportion 10:7) to avoid crystallization. In the following of this paper, each displacement, each spatial coordinate and each length is expressed in terms of the diameter of smallest grains $d_{g}$. At the beginning of the experiment, both metallic intruders (black 




Figure 1. Picture of the experimental setup showing the two mechanical intruders in the 2D packing. The white dashed square shows the typical area studied to measure the influence of the distance $s$, which is $35 d_{g}$ on the picture.

round plates in Fig. 1) start to be extracted downward at a constant velocity $V$ of $0.05 \mathrm{~mm} . \mathrm{s}^{-1}$ involving a quasi-static evolution of the surrounding media due to the weight of the grains. We keep the same low velocity for all experiments.

During the experiment, we record the evolution of the cell recording images each $5 \mathrm{~s}$. We then measure precisely the position of each grains, and identify their displacement between successive images using a tracking algorithm. We will change the distance $s$ between the intruders, as well as their width $w$, to measure their impact on the reorganization of the media.

\section{Results}

\subsection{Mean displacement field}

We first maintain fixed the position of one intruder while the other is moving down (toward negative values of $y$ ). During this movement, we measure the average vertical displacement of the grains on the horizontal line $y=1$. While the $y$ coordinates of the grain is pointing upward, the vertical displacement are generally in the negative direction, so we always report their opposite values $\gamma$. The resulting curve is plotted twice in the figure $2 \mathrm{a}$, each time at the position of one intruder. Then we compare the experimental vertical displacement observed when the two intruders are moving together at the same speed with the superposition of the previous curves as if there were no correlation (Fig. 2b), for two different initial positions of the pair of intruders. In both cases, the agreement between the black and the colored lines is fairly good, meaning that for such separation ( $s=17.5 d_{g}$ and $35 d_{g}$ ) the grains around one intruder are weakly influenced by the other intruder. On the contrary, for close intruders (Fig. 3a), the mean displacement in the middle of the cell is larger than the one expected from the simple superposition of the displacements from single intruders (fig. 3b). This result shows that at short distance, cooperation must be expected between intruders: the destabilization above one apex must propagate toward the grains around the other intruder.


Figure 2. (a) Superposition of two vertical displacements of the grains along the axe $y=1$, obtained from experiments with one fixed intruder in the cell. Its width is $w=10 d_{g}$ and we duplicated the profiles at the place of the twin intruder for $s=17.5$. (b) Vertical displacements of the grains along the axe $y=1$, when pairs of intruders are moving for $s=17.5 d_{g}$ (yellow dashed line) and $s=35 d_{g}$ (blue dashed line). The solid black lines are the sum of the displacement obtained from a single intruder plotted in $a$.
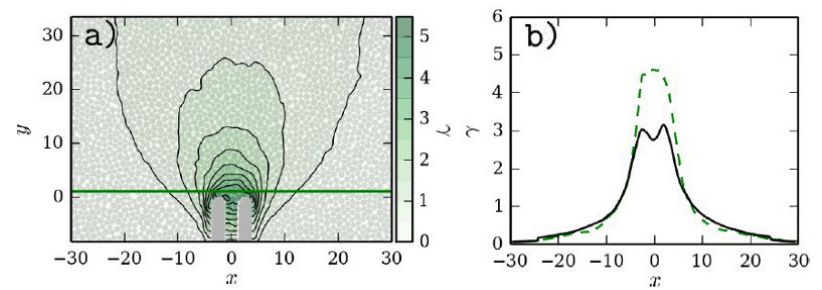

Figure 3. Vertical displacement $\gamma$ between the first and the last image of the grains. (a) Displacement field for two close and narrow intruders of width $w=2.5 d_{g}$ separated by $s=5 d_{g}$. (b) Vertical displacements of the grains along the axe $y=1$ for the close narrow intruders (green dashed line). The solid black line is the sum of the shifted displacements obtained from a single narrow intruder.

\subsection{Avalanche sizes}

To quantify this influence, we first define the size of an avalanche based on the displacement of the grains. Between two consecutive images, we count the number of grains that move at least as much as the intruder. To focus on a relevant area, we restrict this analysis to grains inside the white square of the figure 1, whose right border is aligned with the border of the left intruder. This number $N_{A}$ defines the avalanche size. In the figure 4 , we plot the distribution of the avalanche size for different distance $s$ between the two intruders. First, we observe that the distribution for a single intruder is the asymptotic distribution when the distance increases between the pair of intruders. Second, when the distance is shorter than 15 grain-diameters, the distributions start to deviate and the maximum is shifting toward larger amplitudes, while the probability to observe a size of 0 decreases strongly. This means that the closer are the intruders, the less jammed is the system. Finally, the probability of the large events decreases as a power law with an exponent of -2.6 . 

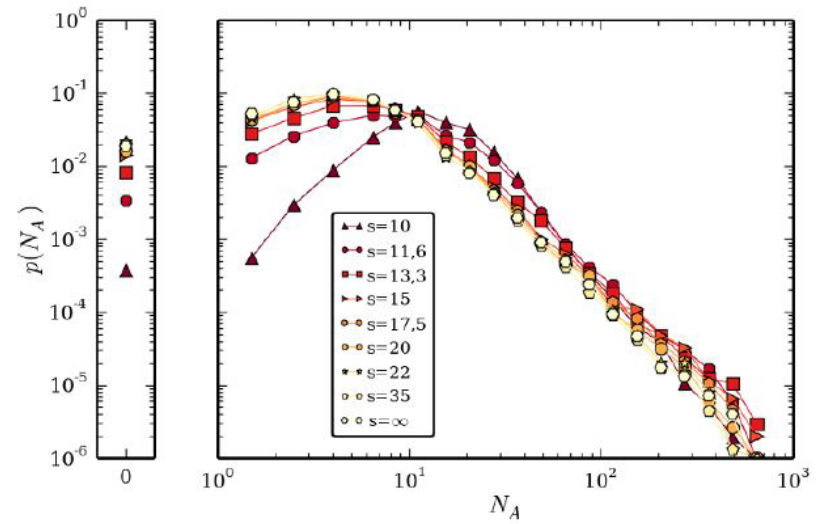

Figure 4. Avalanche size distributions in the white square of the figure 1 for a pair of intruders of width $10 d_{g}$. From the lightest curve to the darkest curve, the distance $s$ decreases. The case of a single intruder is denoted by $s=\infty$.

\subsection{Characteristic lengths}

The data show that a critical length exists to observe a notable influence of a near intruder on the distribution of avalanche size. We measure this effect for different widths of intruder. To compare the results, we plot only the mean avalanche size of the distributions $\left\langle N_{A}\right\rangle$ as a function of the shifted distance $(\hat{s}=s-w)$ for all intruders in figure 5. The sizes for far intruders converges toward the mean size obtained for a single intruder $\left\langle N_{A}\right\rangle_{0}$, as shown by the horizontal lines in the inset of the figure 5, where the typical evolution of the mean size with $s$ is shown for a simulation and an experiment. We then use this value to normalize our data. The data collapse on a single master curve except the experimental data for the narrower intruders. We can fit this curve by an exponential decrease of characteristic length $4 d_{g}$. This results show that the destabilization near an intruder propagates toward the other site and may trigger an event if they are close enough. This situation is similar to the case of the silo with multiple orifices, where the flow through one outlet may unjam the flow of the second outlet [23].

We also note that the very first points at small separation depart from the master curve. This effect is due to the lack of space between the intruders. They behave like a single larger intruder. The increase near the axis $\hat{s}=0$ is then attributed to the increase of the effective size of the intruder. Finally, the green triangle do not align with other points. These points correspond to the narrowest experimental intruder of $2.5 d_{g}$ in width. We did not identify the origin of this difference since the simulation with the same width collapses on the master curve. One explanation may be that for such narrow intruder, the formation of stable arches above the intruder is widely observed. These structures may be more stable in experiments due to friction against the glass walls and lead to a different dynamic.

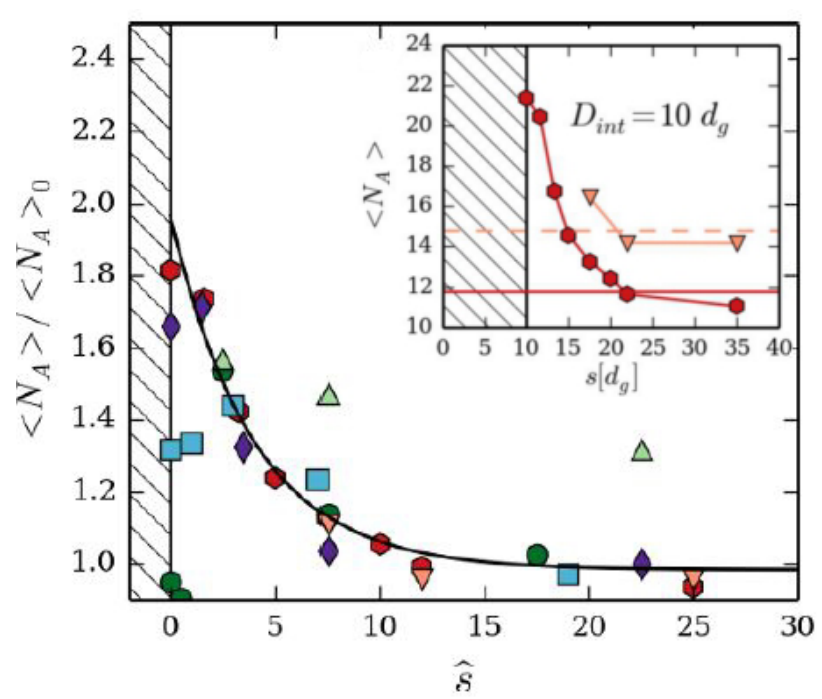

Figure 5. Normalized mean avalanche size as a function of the shifted distance $(\hat{s}=s-w)$ for different widths of intruders $w$ : $10 d_{g}$ (hexagon), $7.5 d_{g}(\diamond), 5 d_{g}$ ( $\square$ )and $2,5 d_{g}(\circ)$ for the simulations and $10 d_{g}(\nabla)$ and 2,5 $d_{g}(\Delta)$ for the experiments. Inset: same data with the horizontal lines representing the measured reference value $<N_{A}>_{0}$ in each cases.

\section{Conclusion}

We measured the behavior of a granular packing around two intruders to mimic two reactive sites, both numerically and experimentally. We have shown the influence of one intruder on the other. The effect increases exponentially when the distance between them decreases. The characteristic length does not depend on the width of the intruder but on the size of the grains in the system. We expect to observe a larger influence if the two sites are not on the same horizontal line, as it would be the case in a real reactive packing. Further works will investigate the role of a vertical gap between intruders and the role of the spatial concentration of reactive sites.

\section{References}

[1] F. Ludewig, N. Vandewalle, S. Dorbolo, M. Pakpour, G. Lumay, Physical Review E 92, 010202 (2015)

[2] B. Turnbull, Physical Review Letters 107, 258001 (2011)

[3] D.M. Kintea, T. Hauk, I.V. Roisman, C. Tropea, Physical Review E 92, 033012 (2015)

[4] Q. Xu, A.V. Orpe, A. Kudrolli, Physical Review E 76, 031302 (2007)

[5] S. Herminghaus, Advances in Physics 54, 221 (2005)

[6] E. Gouillart, M.J. Toplis, J. Grynberg, M.H. Chopinet, E. Sondergard, L. Salvo, M. Suéry, M. Di Michiel, G. Varoquaux, Journal of the American Ceramic Society 95, 1504 (2012)

[7] B. Andreotti, Y. Forterre, O. Pouliquen, Granular media: between fluid and solid (Cambridge University Press, 2013) 
[8] P. Jop, Y. Forterre, O. Pouliquen, Nature 441, 727 (2006)

[9] M. Wyart, Physical Review Letters 109 (2012), arXiv: 1202.0259

[10] N. Mitarai, F. Nori, Advances in Physics 55, 1 (2006)

[11] O. Lame, D. Bellet, M. Di Michiel, D. Bouvard, Nuclear Instruments and Methods in Physics Research Section B: Beam Interactions with Materials and Atoms 200, 287 (2003)

[12] P.z. Wong, J. Koplik, J.P. Tomanic, Physical Review B 30, 6606 (1984)

[13] S. Dorbolo, F. Ludewig, N. Vandewalle, C. Laroche, Physical Review E 85, 051310 (2012)

[14] E. Kolb, P. Cixous, N. Gaudouen, T. Darnige, Physical Review E 87, 032207 (2013)

[15] C. Coulais, A. Seguin, O. Dauchot, Physical Review Letters 113, 198001 (2014)
[16] E. Hamm, F. Melo, Europhysics Letters (EPL) 73, 356 (2006)

[17] A. Seguin, C. Coulais, F. Martinez, Y. Bertho, P. Gondret, Physical Review E 93, 012904 (2016)

[18] A. Seguin, Y. Bertho, P. Gondret, J. Crassous, Physical Review Letters 107, 048001 (2011)

[19] E. Kolb, P. Cixous, J.C. Charmet, Granular Matter 16, 223 (2014)

[20] J. Choi, A. Kudrolli, M.Z. Bazant, Journal of Physics: Condensed Matter 17, S2533 (2005)

[21] A. Janda, I. Zuriguel, A. Garcimartín, L.A. Pugnaloni, D. Maza, EPL (Europhysics Letters) 84, 44002 (2008)

[22] F. Melo, F. Vivanco, C. Fuentes, V. Apablaza, International Journal of Rock Mechanics and Mining Sciences 45, 248 (2008)

[23] A. Kunte, P. Doshi, A.V. Orpe, Physical Review E 90, 020201 (2014) 